\title{
Mehr als ein Sportereignis: Die modernen Olympischen Spiele - von Athen 1896 bis München 1972
}

\author{
Ansgar Molzberger
}

\section{Abstract}

Werte, Wettkampf, Weltereignis - die Olympischen Spiele sind mehr als ein internationales Sportfest. Vielmehr liegt ihnen eine spezifische Sinngebung zugrunde, die der französische Baron Pierre de Coubertin (1863-1937) Ende des 19. Jahrhunderts definiert und fortan - zusammen mit seinen Mitstreitern im IOC - weiterentwickelt hat. Bereits zu seinen Lebzeiten ließen sich nicht mehr nur herausragende sportliche Leistungen mit dem olympischen Motto „citius, altius, fortius“ (seit 2021: „citius, altius, fortius, communis“) in Verbindung bringen, auch die Olympischen Spiele selbst wurden immer größer und damit zum globalen Kulturphänomen, das den jeweils herrschenden Zeitgeist spiegelt - und selbst Geschichte schreibt. In der Auseinandersetzung mit der Entwicklung der Olympischen Spiele von ihren Anfängen bis zu den Herausforderungen der heutigen Zeit, das Weltereignis Olympische Spiele auch weiterhin realisieren zu können, liegen Bildungspotenziale, die weit über das alle vier Jahre stattfindende Sportgeschehen in der olympischen Arena hinausgehen.

\section{Einleitung}

Die Olympischen Spiele sind mehr als eine Weltmeisterschaft in verschiedenen Sportarten. Vielmehr liegt den Spielen eine spezifische, pädagogisch inspirierte Sinngebung zugrunde, darüber hinaus stehen sie immer auch in Wechselwirkung mit dem gesellschaftlichen, politischen und kulturellen Zeitgeist und erfahren als Weltereignis größtmögliche Aufmerksamkeit. Vor diesem Hintergrund stellen sie nicht nur einen attraktiven Forschungsgegenstand dar, aus der Auseinandersetzung mit ihnen lassen sich auch vielfältige Bildungspotenziale ableiten.

Entscheidende Entwicklungen in der Geschichte der Olympischen Bewegung sollen im vorliegenden Beitrag in komprimierter Form aufge- 
zeigt werden, vornehmlich aus einer deutschen Perspektive. Dies bringt mit sich, dass die an Berlin vergebenen Spiele von 1916 - die dem Ersten Weltkrieg zum Opfer fielen - sowie die Olympischen (Winter-) Spiele von 1936 in Garmisch-Partenkirchen und Berlin ausführlicher behandelt werden. Weiterhin die Spiele 1972 in München - bis heute die letztmalige Austragung Olympischer Spiele in einer deutschen Stadt.

1972 bildet daher auch die Zäsur für die zeitliche Eingrenzung dieses Artikels - zumal jüngere, die Olympische Bewegung prägende Phänomene wie die großen internationalen Boykotte sowie die Ökonomisierung und Mediatisierung der Spiele in weiteren Beiträgen zu diesem Buch gesondert behandelt werden.

\section{Zur Vorgeschichte}

Der französische Baron Pierre de Coubertin (1863-1937) war nicht der erste, der die Idee einer modernen „Wiedergeburt“ der Olympischen Spiele - als sehr freie Interpretation des antiken Vorbilds hatte. Bereits ab dem 17. Jahrhundert waren in England und später auch in Deutschland, Schweden und Frankreich einzelne sogenannte „Olympische Spiele“ veranstaltet worden. Diese waren jedoch vor allem regionaler oder nationaler Natur, zudem wurden sie bis auf wenige Ausnahmen nach einzelnen Austragungen wieder eingestellt. Als griechische Vorläuferveranstaltung sind zudem die durch eine Stiftung des wohlhabenden Kaufmanns Evangelos Zappas (1800-1865) ins Leben gerufenen „Olympien“ zu nennen, eine Kombination von sportlichen Wettkämpfen und einer Leistungsschau der heimischen Industrie und Agrarwirtschaft. Im Zeitraum von 1859 bis 1889 wurden die „Olympien“ viermal in Athen ausgetragen (Decker, 1996).

Im späten 19. Jahrhundert wurde Coubertin, der einzelne der „Vorolympischen Spiele“ kannte, dann aber vor dem zeitgeschichtlichen Hintergrund der neuhumanistischen Auseinandersetzung mit der Antike, diverser Reformbewegungen und einer internationalen Friedensbewegung zum Initiator der modernen Olympischen Bewegung. Als Anhänger des angloamerikanischen Sports und Pädagoge aus Leidenschaft hatte Coubertin $1894 \mathrm{zu}$ einem internationalen Sportkongress, der ursprünglich die Amateurfrage zum Hauptthema haben sollte, an die Pariser Sorbonne eingeladen. Im finalen Programmheft lautete der Tagungstitel dann jedoch „Congrès International de Paris pour le Rétablissement des Jeux Olympiques“. Bei der Schlusssitzung am 23. Juni 1894 entschieden die Delegierten, die Olympischen Spiele in moderner Form wieder aufleben 
zu lassen (Müller, 1994) - vor diesem Hintergrund wird der 23. Juni heute international als „Olympic Day“ gefeiert. Die Premiere sollte 1896 in Athen, die zweiten Olympischen Spiele 1900 in Paris stattfinden.

Von Beginn an war es das Bestreben Coubertins, den olympischen Sport - der Amateuren vorbehalten bleiben sollte - mit einem besonderen Sinngehalt zu versehen. In "seinen“ Olympischen Spielen sah er die Bühne für eine Erziehungsform, die die harmonische Entwicklung körperlicher, geistiger und moralischer Eigenschaften zum Ziel hatte. Der sportliche Wettkampf und das Streben nach persönlicher Bestleistung selbst der absolute Rekord wurde nicht ausgeschlossen - standen hierbei im Zentrum. Zudem sollte der fair geführte, länder- und kulturübergreifende Wettstreit der Völkerverständigung dienen und so einen Beitrag zur Friedensförderung leisten. Darüber hinaus war es Coubertins Bestreben, die Olympischen Spiele besonders würdevoll zu veranstalten, auch äußerlich sollte das Fest mehr darstellen als internationale Sportmeisterschaften.

Für die Leitung der modernen Olympischen Bewegung wurde 1894 ein Gremium ins Leben gerufen, das sich ab ca. 1898 Comité International Olympique beziehungsweise International Olympic Committee (IOC) nannte (Kluge, 1997).

\section{Athen 1896 - die olympische Premiere}

Am 6. April 1896 wurde in Athen durch den griechischen König Georg I. (1845-1913, von 1863 bis 1913 König der Hellenen) erstmals das Fest eröffnet, das heute das weltweit größte Sportereignis darstellt. Als zentraler Austragungsort diente das ursprünglich im 5. Jahrhundert v. Chr. erbaute Panathenäische Stadion, das mit Hilfe einer großzügigen Spende des griechischen Geschäftsmanns Georgios Averoff (1818-1899) für die ersten modernen Olympischen Spiele rekonstruiert worden war.

Bis zum Abschluss der Veranstaltung am 15. April 1896 maßen sich gut 250 Athleten - Frauen waren nicht zugelassen - in den Sportarten Fechten, Gewichtheben, Leichtathletik, Radsport, Ringen, Schießen, Schwimmen, Tennis und Turnen. Rudern war 1896 ebenfalls vorgesehen, die Wettbewerbe konnten wegen schlechter Witterung jedoch nicht ausgetragen werden.

Auch knapp zwei Dutzend deutsche Athleten waren in Athen am Start, darunter "wilde“ Turner, die gegen den Willen ihres Verbands, der Deutschen Turnerschaft (DT), nach Griechenland gereist waren. Die strikte Ablehnung des englisch geprägten Wettkampfsports, wie er bei den Olympischen Spielen unter dem Motto „citius, altius, fortius“ von Beginn 
an praktiziert wurde, bestimmte in dieser Zeit noch die Haltung der sich auf Friedrich Ludwig Jahn (1778-1852) berufenden Turn-Traditionalisten. Nachdrücklich für die Beteiligung Deutschlands an den Olympischen Spielen hatte sich hingegen der Berliner Chemiker Willibald Gebhardt (1861-1921) ausgesprochen, der im Zuge der Athener Spiele als erster Deutscher ins Internationale Olympische Komitee berufen wurde (Naul, 1999).

1896 zeigten sich dann in der griechischen Hauptstadt ausgerechnet jene „wilden“ Turner besonders erfolgreich, die offen waren für die Idee des olympischen (Leistungs-) Sports. Darunter der Berliner Carl Schuhmann (1869-1946), der zum deutschen Star der Spiele avancierte, indem er nicht nur drei Turnwettbewerbe gewann, sondern auch die Konkurrenz im Ringen. Mit Goldmedaillen wurde er für seine Olympiasiege indes nicht belohnt: Aus Kostengründen erhielten die Gewinner bei den Athener Spielen "nur" silberne Medaillen, ein Diplom und - in Anlehnung an die antiken Olympischen Spiele - einen Ölbaum-Zweig. Die Zweitplatzierten erhielten eine kupferne Medaille, ein Diplom und einen Lorbeerzweig, die Drittplatzierten gingen leer aus.

Den Höhepunkt der Athener Veranstaltung stellte der Marathonlauf dar; ein Wettbewerb, der in Anlehnung an die antike Legende des Botenlaufs von Marathon nach Athen für das olympische Fest ins Leben gerufen worden war und zum Publikumsmagneten wurde. Zur Begeisterung der Ausrichter siegte mit Spiridon Louis (1873-1940) ein Grieche. Für die Strecke von ca. $40 \mathrm{~km}$ - erst 1921 legte der Internationale Leichtathletikverband (World Athletics, vormals: IAAF) die bis heute gültige Distanz von 42,195 km fest - benötigte er knapp drei Stunden.

\section{Weltausstellungen und Olympische Spiele}

Nach Abschluss der Athener Spiele übernahm Pierre de Coubertin das Amt des IOC-Präsidenten - gemäß ursprünglicher Vereinbarung, dass der amtierende Präsident aus dem Heimatland der nächsten Olympiastadt kommen sollte, hatte von 1894 bis 1986 der griechische Literat Dimitrios Vikelas (1835-1908) als Präsident fungiert.

Zunächst musste man Kompromisse eingehen, um die Olympischen Spiele am Leben zu halten. So wurden sie in den folgenden Jahren in (Welt-) Ausstellungen eingebunden, da sie als autonome Veranstaltung nicht stark genug schienen. Die fehlende Eigenständigkeit des olympischen Fests wurde hierfür billigend in Kauf genommen, insbesondere die Spiele von Paris (1900) und St. Louis (1904) erlebten viele Zeitgenossen 
lediglich als Programmpunkt der dort stattfindenden Weltausstellungen. Auch die Olympischen Spiele von London (1908) waren Teil einer derartigen Schau, der internationalen „Franco-British Exhibition“.

Eigenständigkeit bewies Coubertin hingegen bei der Führung der Olympischen Bewegung. So amtierte er entgegen der zuvor genannten Regelung auch nach 1900 als IOC-Präsident und trat erst 1925 zurück. Selbstbewusstsein zeigten auch die Frauen, indem sie sich ab 1900 mehr und mehr Startplätze bei den Olympischen Spielen erstritten, zunächst im Tennis und Golf, 1912 dann im Schwimmen, 1928 in der Leichtathletik.

Das deutsche Engagement in der Olympischen Bewegung festigte sich 1904, als mit dem Deutschen Reichsausschuss für Olympische Spiele (DRAfOS) ein erstes permanentes Nationales Olympisches Komitee ins Leben gerufen wurde, das sich fortan um die deutschen Olympiateilnahmen kümmern sollte (Langenfeld, 1999). Als Besonderheit in der Geschichte hatte man auch im „Nicht-Olympiajahr“ 1906 eine solche Aufgabe: Der Erfolg von 1896 hatte die Griechen motiviert, die Olympischen Spiele auf Dauer zu beanspruchen. Dies stand jedoch im Gegensatz zu Coubertins Idee von internationalen Spielen mit wechselnden Austragungsorten. Gegen seinen Willen, aber im Einvernehmen mit der Mehrheit der anderen IOC-Mitglieder, luden die Griechen dennoch für 1906 - in der Mitte der III. Olympiade - zum zehnjährigen Jubiläum der ersten Athener Spiele in die griechische Hauptstadt ein. Die „2. Internationalen Olympischen Spiele“, so der offizielle Titel der Veranstaltung, waren ein voller Erfolg. Solche „Zwischenspiele“ fanden danach aber nie wieder statt. Zu einer Art Erweckungserlebnis wurde das Fest für den jungen deutschen Sportjournalisten und -funktionär Carl Diem (1882-1962) - der Einsatz für die Olympische Idee wurde für Diem, der in den Folgejahren zum einflussreichsten deutschen Sportgestalter avancierte, zu einer Lebensaufgabe.

\section{Stockholm 1912 - der Durchbruch}

Als Durchbruch für die Olympische Bewegung gelten die 1912 in Stockholm ausgetragenen Olympischen Spiele. Diese waren nicht nur eine eigenständige und kompakt arrangierte Festveranstaltung, sie stellten auch die Premiere für eine Reihe von Neuerungen dar, die zum festen Bestandteil kommender Spiele wurden. Hierzu gehörten zwei Wettkampfkreationen Coubertins: Zum einen der Moderne Fünfkampf (Schießen, Schwimmen, Fechten, Reiten und Querfeldeinlauf) als „Weihe für den vollkommenen Athleten“ (Coubertin, 1931). Zum anderen die Olympi- 
schen Kunstwettbewerbe, die auf nachdrückliche Forderung Coubertins - und gegen den Willen der schwedischen Organisatoren, die im „sportlichen" Vergleich von Kunstwerken keinen Sinn sahen - ins offizielle Wettkampfprogramm aufgenommen wurden. Die angestrebte „Hochzeit von Muskel und Geist", wie Coubertin es später in seinen Memoiren nannte (Coubertin, 1931), sollte „seine“ Spiele mit der Wissenschaft und der Kunst verbinden. Bis 1948 konnten Künstler*innen in den Kategorien Architektur, Bildhauerei, Literatur, Malerei und Musik ebenso Medaillen erringen wie die Athlet*innen, ehe die bei vielen Sportfunktionären unbeliebten Wettbewerbe aus dem Programm gestrichen wurden.

Das Stockholmer Fest zeigte der Welt jedoch nicht nur das positive Potenzial der Olympischen Bewegung, die aufgetretenen Probleme bei der Planung und Durchführung der sportlichen Wettkämpfe stellten zudem einen Impuls für die dringend notwendige Standardisierung des Sports dar (Molzberger, 2012) - bis dahin hatten nur wenige internationale Fachverbände existiert, die Veranstalter Olympischer Spiele hatten eigene Regelwerke erstellen und kommunizieren müssen. Diese negativen Erfahrungen führten zu Reformbestrebungen, insbesondere die Gründung des Internationalen Leichtathletik-Verbands IAAF (heute: World Athletics) 1912/13 als Reaktion auf die in der schwedischen Hauptstadt erneut zutage getretenen Schwierigkeiten bedeutete einen Meilenstein für das Regelwerk der olympischen Kernsportart Leichtathletik und damit einen wichtigen Schritt in Richtung „Fair Play“ im Sport. 
Abbildung 1: Anmeldeformular (Leichtathletik/Mannschaftskonkurrenzen) für die Olympischen Spiele 1912 in Stockholm. Mit der Unterschrift hatten die Athleten ihren Amateurstatus zu versichern.

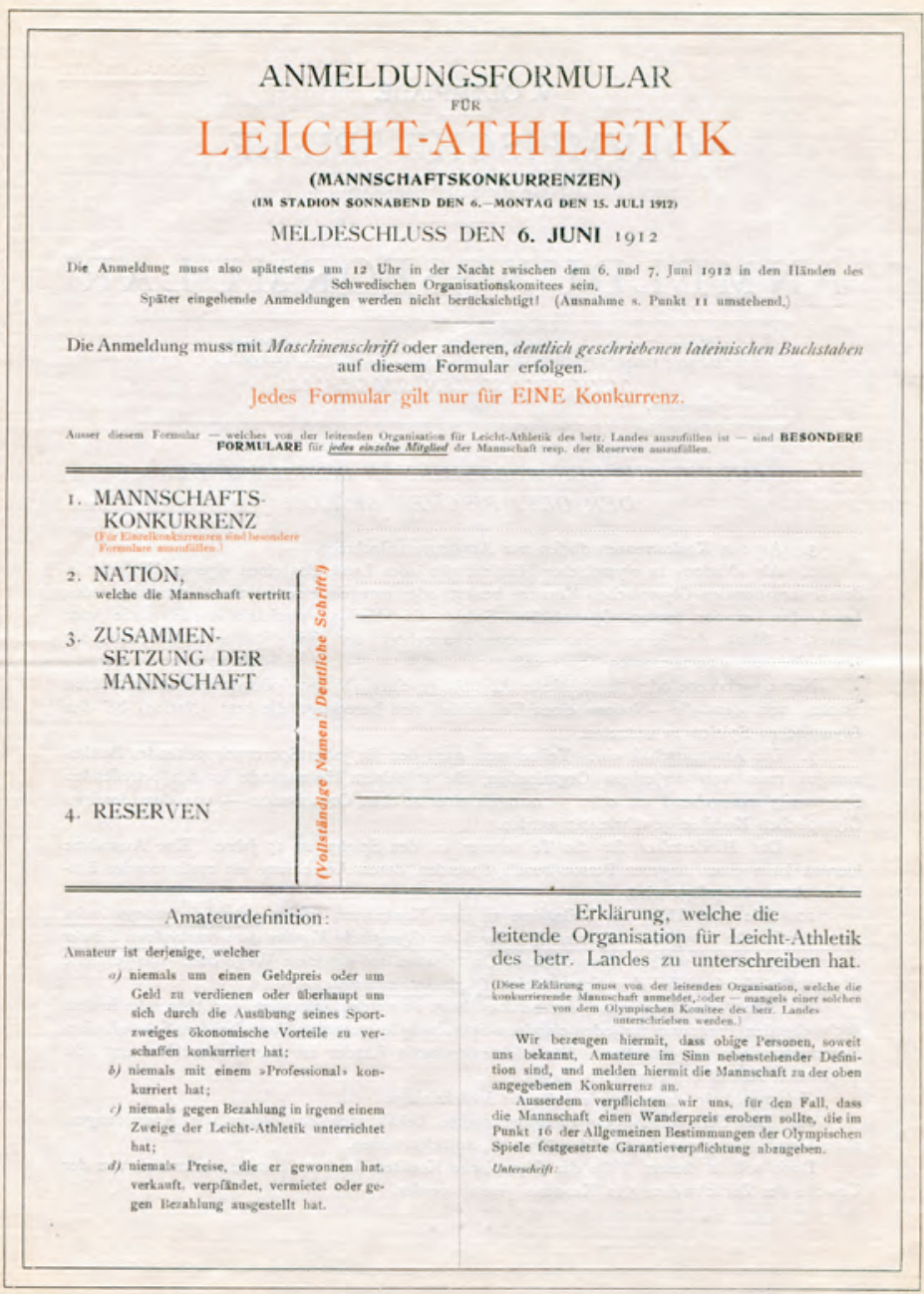

Wenden

(Carl und Liselott Diem-Archiv/Zentrum für Olympische Studien, Deutsche Sporthochschule Köln) 
Die nun zunehmend gefestigte Olympische Bewegung feierte 1914 beim Olympischen Kongress in Paris ihren zwanzigsten Geburtstag. Als "Geschenk" brachte Coubertin eine Kreation mit, die er - aus einer Künstlerfamilie stammend - ein Jahr zuvor selbst geschaffen hatte: die olympische Fahne mit den fünf farbigen Ringen.

\section{Berlin 1916 - Opfer des Kriegs}

Die Freude über den runden Geburtstag währte jedoch nicht lange, der im Sommer 1914 ausgebrochene Erste Weltkrieg wurde zur Existenz bedrohenden Zäsur. Dem Völker verbindenden Gedanken der Olympischen Bewegung zum Trotz hatten bereits in den Vorjahren nationale Autonomiebestrebungen immer wieder für Konflikte auf der olympischen Bühne gesorgt, für die Forderungen von Delegationen aus Böhmen, Ungarn oder Finnland nach eigenständigem Auftritt trotz fehlender staatlicher Souveränität hatte Coubertin den Begriff der "Sportlichen Geografie“ geprägt. Solche Konfliktfälle hatten jedoch „nur“ zu sportdiplomatischen Auseinandersetzungen geführt, nun aber entlud sich die in vielen europäischen Ländern über mehrere Jahre national aufgeheizte Stimmung auf den Schlachtfeldern des Ersten Weltkriegs.

Für die deutschen Olympiavertreter brachte der Krieg auch einen großen sportlichen Verlust mit sich: Nach mehreren gescheiterten Anläufen, Berlin zum Austragungsort der Olympischen Spiele zu machen, hatte das IOC 1912 das Weltsportfest von 1916 an die deutsche Hauptstadt vergeben und den Deutschen Reichsausschuss für Olympische Spiele mit der Organisation beauftragt. Das „Unternehmen Olympia“ stand von nun an ganz oben auf der DRAfOS-Agenda, als Generalsekretär des Organisationskomitees wurde Carl Diem angestellt. Ein zentrales Aufgabenfeld im Rahmen der Vorbereitungen war die Förderung des (Leistungs-) Sports in Deutschland - 1912 in Stockholm hatte das deutsche Team nur fünf Goldmedaillen gewonnen. Gleichzeitig waren die Berliner Organisatoren bestrebt, die Deutsche Turnerschaft nun endlich für die Olympische Bewegung zu gewinnen. Wollte man doch 1916 nicht nur erfolgreich abschneiden, sondern auch imposante, patriotisch geprägte Olympische Spiele unter Einbeziehung aller Turn- und Sportvertreter veranstalten (Molzberger et al., 2018).

Der „Friede zwischen Turnen und Sport“ (Diem, 1914) schien angesichts der heimischen Olympischen Spiele realisierbar, ein positives Signal stellte die Beteiligung von 10.000 Turnern an der Eröffnung des Deutschen Stadions am 8. Juni 1913 dar. In nur 200 Tagen Bauzeit war die 
zentrale Wettkampfstätte für die Spiele von 1916 geschaffen worden. Mit dem IOC einigte man sich auf ein Wettkampfprogramm, das Turnen, Athletik (einschließlich Tauziehen, Ringen und Gewichtheben), Radsport, Schwimmen, Rudern, Lawn Tennis (Rasentennis), Fußball, Golf, Hockey, Fechten, Schießen, den Modernen Fünfkampf und - im Rahmen einer für den Februar 1916 geplanten Wintersportwoche im Schwarzwald - auch Eis- und Skilauf umfassen sollte.

\section{Abbildung 2: Postkarte „Deutsches Stadion, Berlin-Grunewald“, 1913.}

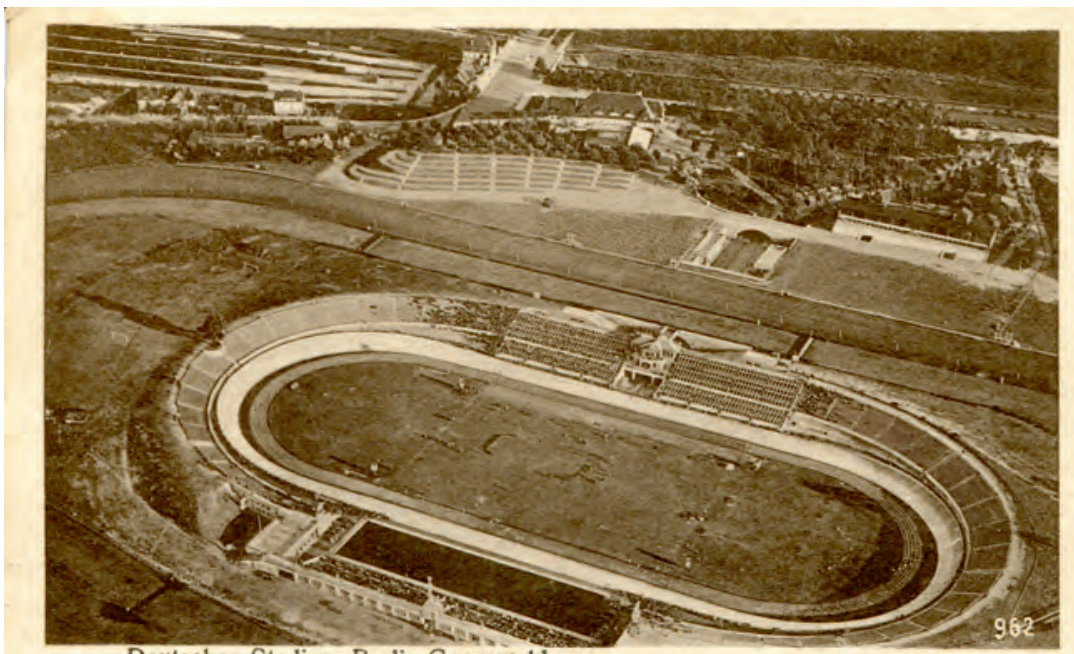

Deutsches Stadion, Berlin-Grunewald

(Carl und Liselott Diem-Archiv/Zentrum für Olympische Studien, Deutsche Sporthochschule Köln)

Im Jahr 1915 sollte ein „nationales Olympia“ als Generalprobe für die Olympischen Spiele von 1916 durchgeführt werden. Zunächst einmal aber wurde das Deutsche Stadion am 27. und 28. Juni 1914 für eine erste große Bewährungsprobe genutzt. Bei den „Olympia-Vorspielen“ in den wichtigsten Sportarten beteiligten sich auch die deutschen Turner mit einem großen Aufgebot. Das am zweiten Tag der Berliner Wettkämpfe in Sarajevo verübte Attentat auf den österreichisch-ungarischen Thronfolger Franz Ferdinand (1863-1914) stellte jedoch den Anfang vom Ende der deutschen Olympiaplanungen dar.

Der Ausbruch des Ersten Weltkriegs am 28. Juli 1914 führte zum Abbruch der sportlichen Vorbereitungen auf die anstehenden Olympischen Spiele. Mit fortlaufender Kriegsdauer zerschlugen sich die anfangs 
noch bestehenden Hoffnungen, das Weltsportfest nach einem baldigen Friedensschluss doch noch durchführen zu können. 1916 wurde der Ausfall der Spiele endgültig zur Gewissheit, auch wenn diese nicht offiziell abgesagt wurden (Molzberger et al., 2018).

Bereits 1915 hatte Pierre de Coubertin das IOC aus Frankreich in das sichere schweizerische Lausanne verlegt - bis heute Sitz des IOC - und das Präsidentenamt kommissarisch an seinen Schweizer Mitstreiter Godefroy de Blonay (1869-1937) übergeben.

\section{Neubeginn nach dem Ersten Weltkrieg}

Kurz nach Ende des Ersten Weltkriegs bemühte sich Coubertin als alter/ neuer IOC-Präsident darum, die Olympische Bewegung zu reaktivieren. Im Jahr 1919 kam das IOC erstmals wieder zusammen. Als Gastgeberstadt für die Olympischen Spiele von 1920 wählte man das belgische Antwerpen. Aufgrund der kurzen Vorbereitungszeit und der noch vorhandenen Kriegsschäden mussten bei der Ausrichtung der Spiele Abstriche in Kauf genommen werden. Deutsche Sportlerinnen und Sportler waren 1920 - und auch noch 1924 - aufgrund der Deutschland zugeschriebenen Kriegsschuld von den Olympischen Spielen ausgeschlossen. Für eine Zusammenkunft aller Athletinnen und Athleten im Sinne der olympischen Idee waren zu diesem Zeitpunkt die Schrecken des Kriegs noch zu präsent.

Im Deutschen Reich wiederum hatte man der Olympischen Bewegung bereits 1917 den Rücken gekehrt. Die Umbenennung des Deutschen Reichsausschusses für Olympische Spiele in Deutscher Reichsausschuss für Leibesübungen (DRA) hatte dies auch auf Ebene des nationalen Dachverbands gezeigt. Mit den sogenannten „Deutschen Kampfspielen“, einer Mehrsparten-Sportveranstaltung, die in Analogie zum internationalen Fest im Vierjahresrhythmus ausgetragen wurde, initiierte der Deutsche Reichsausschuss für Leibesübungen als Ersatz ein „Nationales Olympia“, das in den Jahren 1922, 1926 und 1930 sowohl als Winter- als auch als Sommerausgabe ausgerichtet wurde (Lennartz, 1999).

Vor dem Hintergrund dieser Isolation in der Sportwelt waren dann auch keine Deutschen dabei, als vom 25. Januar bis zum 5. Februar 1924 in den französischen Alpen eine Internationale Wintersportwoche stattfand, bei der knapp 300 Sportler - und einzelne Sportlerinnen - aus insgesamt 16 Nationen im Bobfahren, Eishockey, Eiskunstlauf, Eisschnelllauf und dem Nordischen Skisport um die Medaillen kämpften. Das IOC ernannte diese Wintersportwoche zwei Jahre später rückwirkend zu den 
ersten Olympischen Winterspielen. Eine neue Großveranstaltung war geboren.

Bis dahin hatte das Monopol auf internationalen Winterspielen in Skandinavien gelegen. In Stockholm - und einmal im norwegischen Kristiania, dem späteren Oslo - waren zwischen 1901 und 1926 acht Mal die Nordischen Spiele veranstaltet worden (Jönsson, 2001). Diese hatten nun ausgedient. Fortan gab es alle vier Jahre einen olympischen „Doppelschlag“: Zu Beginn eines Olympiajahres zunächst Winterspiele, im Sommer (oder Herbst) dann die Olympischen Spiele. (Seit 1994 werden die Olympischen Winterspiele bekanntermaßen in einem eigenen, um zwei Jahre versetzten Vierjahres-Zyklus ausgetragen.) (Kluge, 1999)

Im Jahr 1925 folgte ein Machtwechsel an der Spitze des IOC: Coubertin trat als langjähriger Präsident zurück, zu seinem Nachfolger wurde der belgische Graf Henri de Baillet-Latour (1876-1942) gewählt.

\section{Die deutsche „Rückkehr nach Olympia“}

Die Weichen für den Wiedereintritt der Deutschen in die Olympische Bewegung waren bereits 1924 gestellt worden, als mit Theodor Lewald (1860-1947) und Oskar Ruperti (1877-1957) der DRA-Präsident und der Vorsitzende des Deutschen Ruderverbands in das IOC berufen worden waren. Ab 1928 betraten auch deutsche Sportlerinnen und Sportler wieder die olympische Bühne. Bei den Spielen in Amsterdam zeigten sie ein starkes „Comeback“ und gewannen elf Goldmedaillen - nur das US-Team war mit 22 Olympiasiegen erfolgreicher.

Von diesem erfolgreichen Auftreten ermutigt, strebten die deutschen Olympiavertreter nun nach mehr: Berlin, Frankfurt, Köln und Nürnberg trauten sich die Ausrichtung der Olympischen Spiele $1936 \mathrm{zu}$ und bewarben sich auf nationaler Ebene. Die Wahl der Bewerberstadt oblag dem Deutschen Reichsausschuss für Leibesübungen beziehungsweise dem Deutschen Olympischen Ausschuss (DOA), der sich 1926 als DRA-Unterausschuss konstituiert hatte. Die führenden DRA-/DOA-Köpfe, Theodor Lewald und Carl Diem, präferierten die deutsche Hauptstadt, die ja bereits 1916 die Olympischen Spiele hätte ausrichten sollen und nun zum Zuge kommen sollte. Darüber hinaus war Berlin vom IOC mit der Ausrichtung des IX. Olympischen Kongresses im Jahr 1930 betraut worden. Mit der gelungenen Durchführung der Veranstaltung - und dem gleichzeitigen Rückzug der Olympiabewerbungen aus Frankfurt, Köln und Nürnberg konnte man für die deutsche Hauptstadt werben. 1931 war man schließlich am Ziel, das IOC erteilte Berlin den Zuschlag. Zwanzig Jahre nach 
dem kriegsbedingten Ausfall sollten die Olympischen Spiele nun erstmals in einer deutschen Stadt ausgetragen werden (Lennartz, 1999).

Die Auswirkungen der Weltwirtschaftskrise bremsten die Vorbereitungen jedoch merklich, auch die Olympischen (Winter-) Spiele 1932 in Lake Placid und Los Angeles litten darunter, weil mehrere - insbesondere europäische - Nationale Olympische Komitees die hohen Kosten für die Überseereise nicht aufbringen und daher kein oder nur ein kleines Team entsenden konnten.

\section{6 - Olympische Spiele im nationalsozialistischen Deutschland}

Aufgrund der schlechten Wirtschaftslage fielen auch die Pläne für die Vorbereitung und Durchführung der Olympischen Spiele 1936 in Berlin zunächst bescheiden aus. Am 24. Januar 1933 konstituierte sich das „Organisations-Komitee für die XI. Olympiade [sic!] Berlin 1936“ mit Theodor Lewald als Vorsitzendem und Carl Diem als hauptamtlichem Generalsekretär.

Mit der wenige Tage später folgenden Machtübernahme der Nationalsozialisten musste der Traum von „Olympia in Berlin“ aber erneut in Frage gestellt werden, hatten sich die neuen Machthaber in der Weimarer Zeit doch als entschiedene Gegner der Olympischen Bewegung gezeigt. Nach einem Treffen mit Lewald sprach sich der neue Reichskanzler Adolf Hitler (1889-1945) aber bereits im März 1933 öffentlich für die Durchführung der Olympischen Spiele aus, in denen er eine einmalige Chance zur Selbstdarstellung des nationalsozialistischen Deutschlands erkannte.

Die Spiele wurden zur „nationalen Aufgabe“ erklärt und fortan üppig finanziert. Nach den Plänen von Werner March (1894-1976) und Walter March (1898-1969) entstand nach Abriss des für die Spiele von 1916 errichteten Deutschen Stadions das mehr als 130 Hektar große „Reichssportfeld“ mit dem neuen, für 100.000 Zuschauer konzipierten Olympiastadion im Zentrum.

Im Jahr 1934 übernahm Hitler die Schirmherrschaft über das Berliner Organisationskomitee, Vertreter des NS-Staats wie „Reichssportführer“ Hans von Tschammer und Osten (1887-1943) saßen zudem im Vorstand. Aus taktischen Gründen beließ man Lewald - von der NS-Presse zuvor über viele Jahre als „Halbjude“ diffamiert - und Diem im Amt, da man ihre Sachkompetenz benötigte und darüber hinaus gegenüber dem Ausland das Bild eines seriösen (Sport-) Deutschlands erzeugen wollte. Insbesondere Diem als Generalsekretär des Berliner Organisationskomitees konnte dann auch seine zentralen Ideen für die Gestaltung der Olympi- 
schen Spiele realisieren. Neben dem vielfältigen kulturellen Rahmenprogramm mit einem großen Festspiel im Stadion ist hier insbesondere der olympische Fackellauf zu nennen, der 1936 seine Premiere feierte. Mit dem Entzünden des Feuers im antiken Olympia und dem anschließenden Fackelstaffellauf zum Austragungsort der Spiele wurde ein Ritual geschaffen, das zum festen Bestandteil des olympischen Zeremoniells wurde (Bernett \& Teichler, 1999).

\section{Olympische Symbolik}

Zu den Olympischen Symbolen zählen das Logo der Ringe auf weißem Grund, das Olympische Feuer und der Fackelstaffellauf, die Medaillen, die Olympische Hymne, der Olympische Eid oder das Motto "Citius, Altius, Fortius“. Diese Symbole sind beinahe durchgehend Elemente, die erst im Laufe der Entwicklung der modernen Olympischen Spiele Einzug in das Zeremoniell gehalten haben. Einzig das Olympische Feuer kann als Äquivalent zum Feuer verstanden werden, das in der Antike zu Ehren Zeus' entzündet wurde. Informationen zu den einzelnen Symbolen finden Sie in der Broschüre „Basiswissen Olympische Spiele“ (https://olympia-ruft.de/basiswissen/).

Vor der Ausrichtung der Berliner Veranstaltung stand für die deutschen Organisatoren jedoch eine weitere Aufgabe an: die Durchführung der Olympischen Winterspiele 1936 in Garmisch-Partenkirchen. Zum Zeitpunkt der Vergabe der Olympischen Spiele an Berlin hatte man sich im IOC darauf geeinigt, die Entscheidung über den Austragungsort der Winterspiele des Jahres 1936 auf 1933 zu vertagen. Vor allem St. Moritz hatte Interesse bekundet, die Olympischen Winterspiele nach 1928 erneut in der Schweiz auszurichten. Als deutsche Vertreter bewarben sich Garmisch und Partenkirchen - der Zusammenschluss der beiden Gemeinden erfolgte erst 1935.

Im Juni 1933 erhielten die bayerischen Gemeinden den Zuschlag, am 23. August 1933 gründete sich daraufhin das Organisationskomitee für die IV. Olympischen Winterspiele. Diesem stand das deutsche IOCMitglied Karl Ritter von Halt (1891-1964) als Präsident vor, zum Vorstand gehörten neben „Reichssportführer“ von Tschammer und Osten und Staatsvertretern auch Carl Diem als Sportexperte sowie die weiteren deutschen IOCMitglieder Theodor Lewald und Adolf Friedrich zu Mecklenburg (18731969). Für die Errichtung der erforderlichen Sportstätten standen ausreichend Reichsmittel zur Verfügung, bereits 1934 konnten die zentralen Wettkampfstätten eingeweiht werden. 
Vom 6. bis 16. Februar 1936 erlebte die Sportwelt sehr gut organisierte Olympische Winterspiele. Die Wettkämpfe im Nordischen Skisport, auf dem Eis und - als olympische Premiere - im Alpinen Skisport, an denen mehr als 750 Sportlerinnen und Sportler aus insgesamt 28 Ländern teilnahmen, begeisterten über 500.000 Zuschauer"innen. Mit sieben Gold, fünf Silber und drei Bronzemedaillen schnitt das norwegische Team am besten ab, gefolgt von den Deutschen und Schweden. Sowohl die Organisatoren als auch große Teile der Sportwelt, insbesondere das IOC, verbuchten die IV. Olympischen Winterspiele nach ihrem Abschluss als gelungene Veranstaltung.

\section{Abbildung 3: Der Norweger Birger Ruud (1911-1998) beim Skispringen} („,Spezialsprunglauf") von der Großen Schanze am letzten Tag der Olympischen Winterspiele 1936. Mit Weiten von $75 m$ und 74,5m sowie guten Haltungsnoten sicherte sich Ruud seinen zweiten Olympiasieg nach 1932.

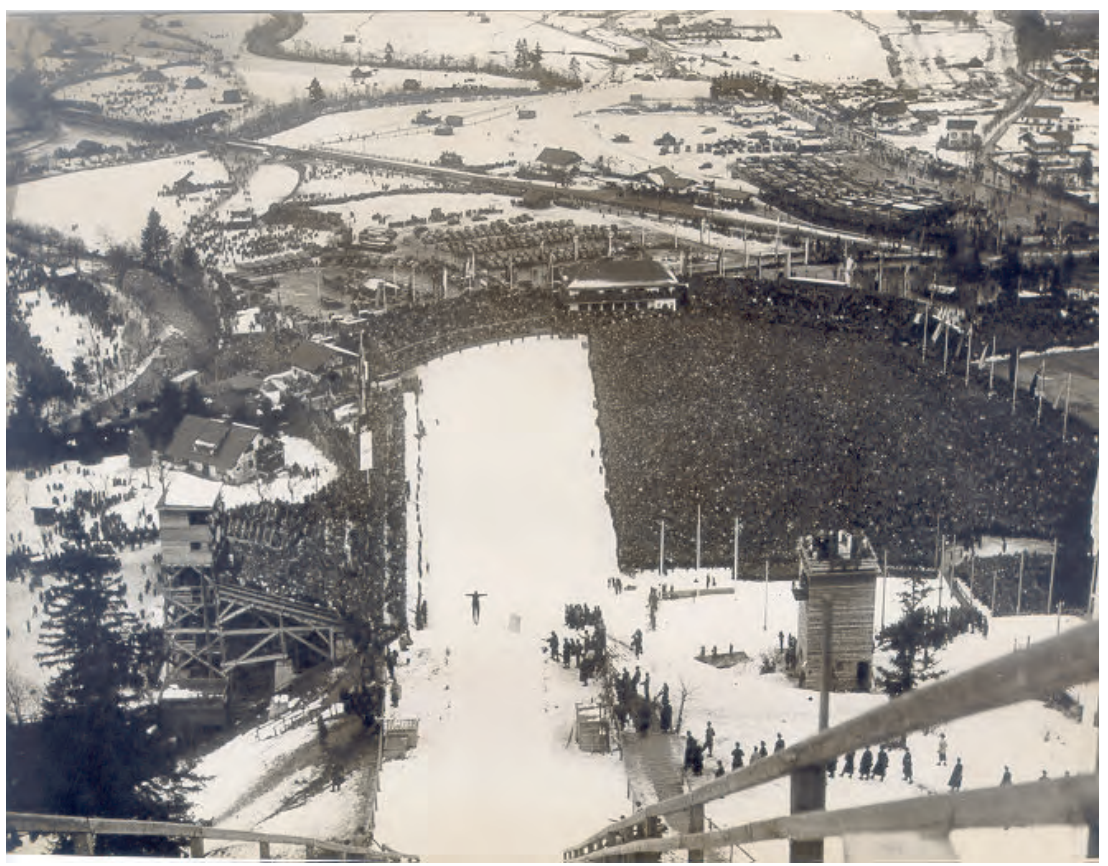

(Carl und Liselott Diem-Archiv/Zentrum für Olympische Studien, Deutsche Sporthochschule Köln) 
Die aggressiv antisemitische Politik der Nationalsozialisten hatte hingegen frühzeitig starke internationale Proteste hervorgerufen, insbesondere in den USA, und vor allem mit Blick auf die - größeren und bedeutsameren - Olympischen (Sommer-) Spiele 1936 war eine Boykottdiskussion entstanden. Das IOC forderte eine Garantie des Organisationskomitees 1936, die olympischen Regeln einzuhalten. Diese wurde 1934 durch die deutschen IOC-Mitglieder von Halt und Lewald gegeben. Als nach der Verabschiedung der antisemitischen „Nürnberger Gesetze“ 1935 der internationale Protest gegen das deutsche Regime nochmals anwuchs, erwirkte der beunruhigte IOC-Präsident Henri de Baillet-Latour ein Treffen mit Hitler, der ihm ebenfalls die Einhaltung der olympischen Regeln bei den Spielen zusicherte. Nach einem knappen Votum des US-amerikanischen Olympischen Komitees im Dezember 1935 für die Teilnahme an den Olympischen Spielen 1936 - vor allem der einflussreiche Sportfunktionär und spätere IOC-Präsident Avery Brundage (1887-1975) hatte sich für die Entsendung des US-Teams nach Berlin ausgesprochen - scheiterte die internationale Boykottbewegung (Molzberger et al., 2018). 
Abbildung 4: Titelblatt der US-amerikanischen Programmschrift „Preserve the Olympic Ideal“, 1935. Das „Committee on Fair Play in Sports", dem mehrere prominente US-Athleten und Funktionäre angehörten, sprach sich für einen Boykott der Olympischen Spiele 1936 aus, scheiterte jedoch mit seinem Anliegen.

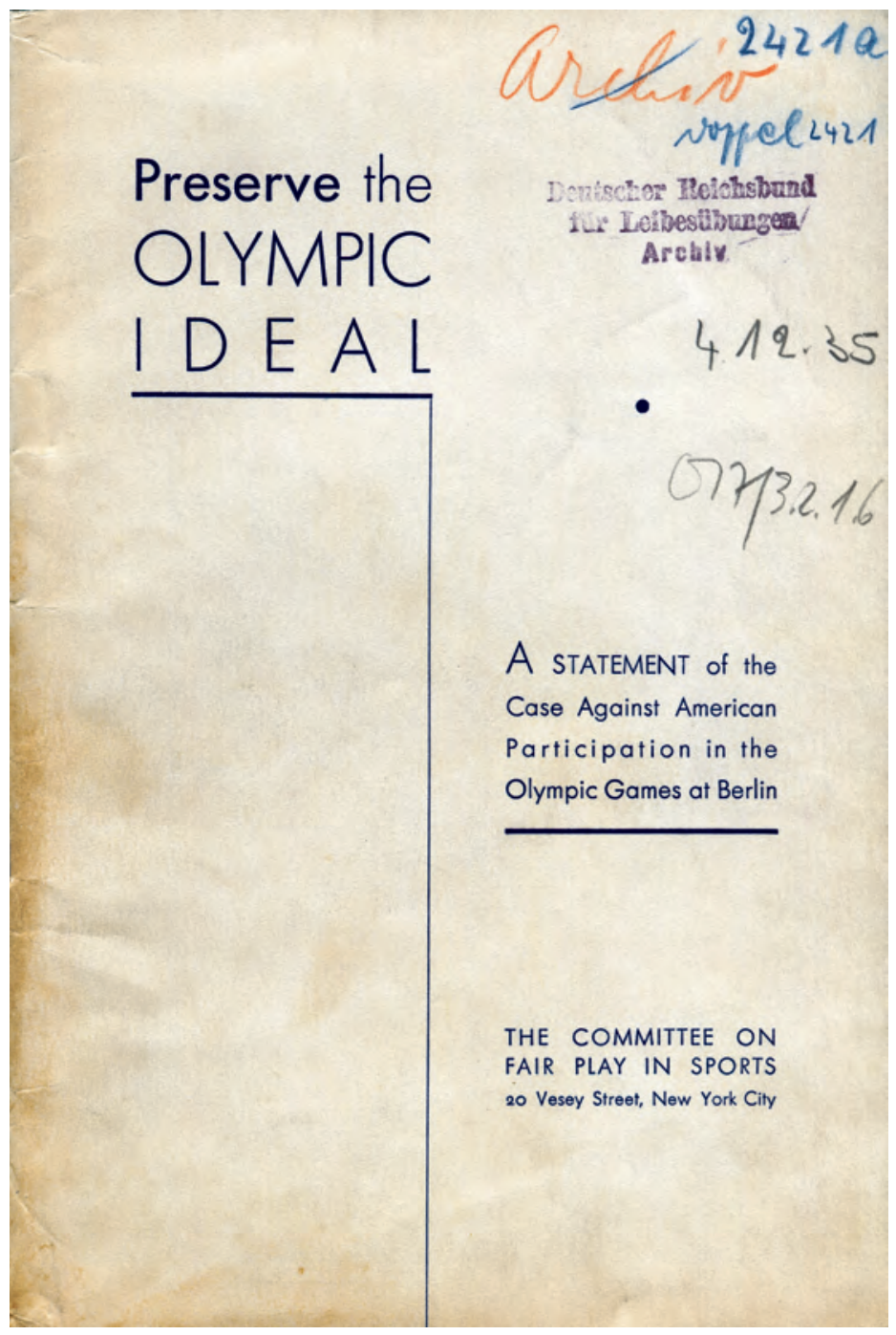

(Carl und Liselott Diem-Archiv/Zentrum für Olympische Studien, Deutsche Sporthochschule Köln) 
Um das wahre Gesicht des „Dritten Reichs“ zu verschleiern, untersagten die Machthaber während der Olympischen (Winter-) Spiele antisemitische und rassistische Anfeindungen in der deutschen Presse und im öffentlichen Raum. Mit dem Eishockeyspieler Rudolf „Rudi“ Ball (19101975) und Helene Mayer (1910-1953), Fecht-Olympiasiegerin 1928 in Amsterdam (Florett), wurden zudem bewusst ein Athlet und eine Athletin für die deutsche Olympiamannschaft nominiert, die laut NS-Jargon als „Halbjuden“ galten.

Mit den genannten Maßnahmen sollte das Bild eines die Menschenrechte achtenden, friedliebenden Deutschlands erzeugt werden. Die Realität der NS-Diktatur sollte ausländischen Beobachtern hingegen weitgehend verborgen bleiben.

Abbildung 5: Eröffnungsfeier der Olympischen Spiele in Berlin am 1. August 1936 - die Friedenssymbolik der auffliegenden Tauben war damals ein bereits etablierter Bestandteil des olympischen Zeremoniells.

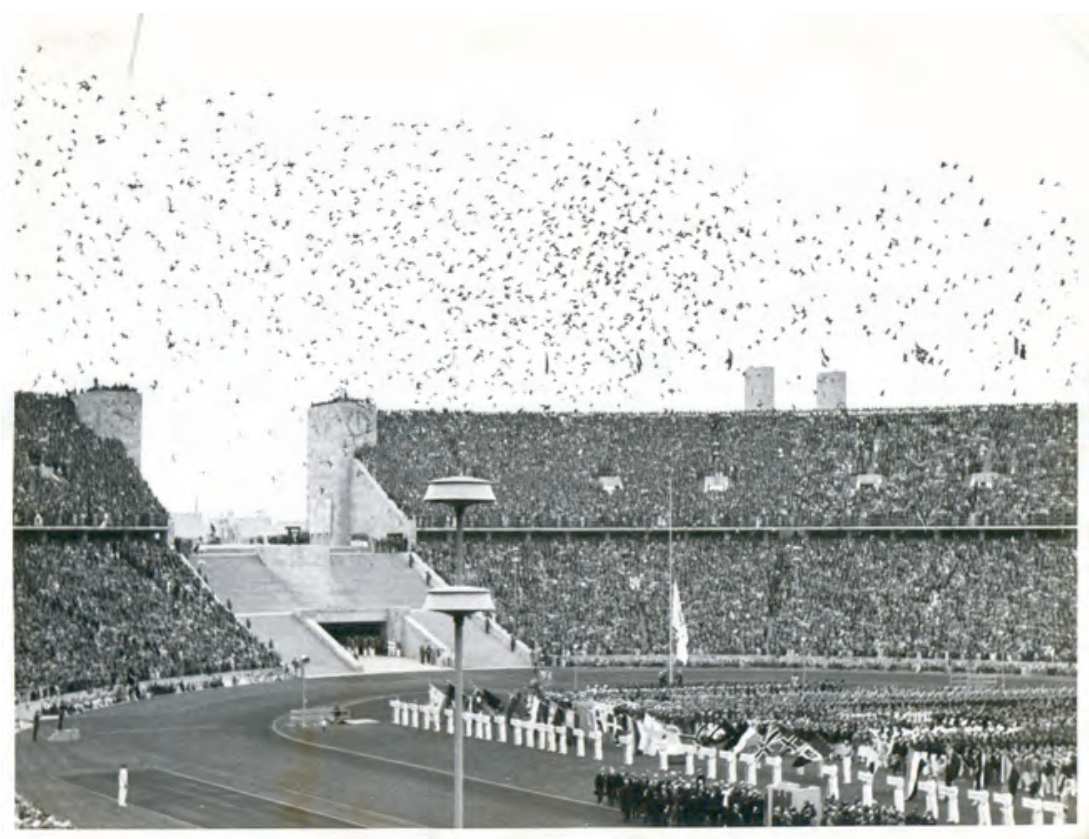

(Carl und Liselott Diem-Archiv/Zentrum für Olympische Studien, Deutsche Sporthochschule Köln) 
An den Olympischen Spielen in Berlin vom 1. bis zum 16. August 1936 nahmen fast 4.000 Athletinnen und Athleten aus 49 Ländern - mehr als jemals zuvor - teil. Spitzenleistungen wie die des US-amerikanischen, vierfachen Olympiasiegers Jesse Owens (1913-1980) und spannende Wettkämpfe begeisterten das Publikum. Zur Freude des heimischen Publikums gewann das deutsche Team die meisten Medaillen.

\section{Abbildung 6: Umringt von Autogrammjägern: Jesse Owens im Berliner Olympiastadion nach seinem Weltrekord-Zwischenlauf $(10,2 s)$ über 100m am 2. August 1936. Das Finale am nächsten Tag gewann Owens mit einer Zeit von 10,3s.}

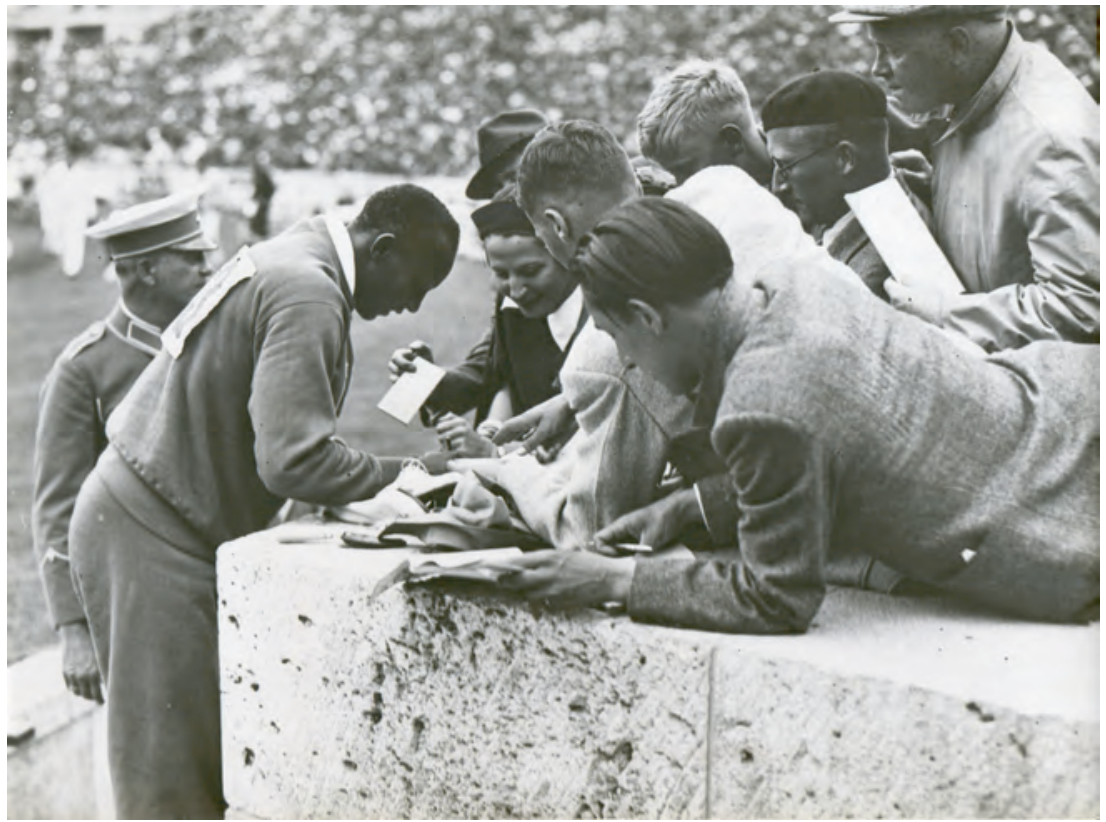

(Carl und Liselott Diem-Archiv/Zentrum für Olympische Studien, Deutsche Sporthochschule Köln)

Darüber hinaus setzten die perfekte Organisation, hervorragend konzipierte Wettkampfstätten, eine beeindruckende Inszenierung und nicht zuletzt modernster Medieneinsatz - das Fernsehen feierte Premiere bei den Olympischen Spielen und die Regisseurin Leni Riefenstahl (1902-2003) setzte die Berliner Veranstaltung mit ihrem bis heute intensiv diskutierten 
Olympiafilm cineastisch in Szene - neue Maßstäbe. Große Teile der Sportwelt erlagen der Illusion eines friedliebenden Deutschlands.

Dennoch zeigten internationale Pressestimmen, dass es sehr wohl Menschen gab, die das von den Machthabern inszenierte Trugbild entlarvten und über die wahren Zustände im nationalsozialistischen Deutschland des Jahres 1936 kritisch berichteten.

Gleichwohl hinterließ die Ausrichtung der Spiele auch positive Spuren: Als drei Jahre später die Planungen für die Austragung der Olympischen Winterspiele 1940 sowohl im zunächst gewählten Sapporo als auch in der Ersatzkandidatenstadt St. Moritz scheiterten, vergab das IOC im Juni 1939 - der aggressiv-expansiven NS-Politik der Vorjahre zum Trotz - die Spiele kurzfristig erneut an Garmisch-Partenkirchen. Ausschlaggebend für diese Entscheidung war, dass sämtliche benötigten Sportstätten vorhanden waren. Am 1. Juli 1939 gründete sich daraufhin das Organisationskomitee. Karl Ritter von Halt stand diesem erneut als Präsident vor, Carl Diem fungierte als Generalsekretär.

Doch wenige Wochen später gaben die Organisatoren nach dem deutschen Überfall auf Polen am 1. September 1939 und dem Ausbruch des Zweiten Weltkriegs die Gastgeberrolle im November 1939 an das IOC zurück. Die Olympischen Winterspiele und die Olympischen Spiele 1940 und 1944 konnten kriegsbedingt nicht ausgetragen werden (Molzberger et al., 2018).

\section{Die Olympische Bewegung nach dem Zweiten Weltkrieg - „Querelle d'allemand"}

Bereits 1942 war IOC-Präsident Henri de Baillet-Latour gestorben, seinen Posten hatte der Schwede J. Sigfrid Edström (1870-1964) kommissarisch übernommen. Mit Kriegsende 1945 setzte sich Edström, der ein Jahr später auch offiziell zum IOC-Präsidenten gewählt wurde, für die Fortsetzung der Olympischen Spiele ein. Im Jahr 1946 erhielten St. Moritz (Winter) und London (Sommer) den Zuschlag für die Ausrichtung der Olympischen Spiele 1948.

Eine deutsche Mannschaft war 1948 noch nicht wieder am Start - zu präsent waren die NS-Gräueltaten in der internationalen Staatengemeinschaft, und im von den Siegermächten des Zweiten Weltkriegs besetzten Deutschland fehlte es zudem noch an den notwendigen Sportstrukturen. Darüber hinaus verfolgte man in der Sowjetischen Besatzungszone die zu dieser Zeit noch geltende politische Linie der UdSSR, wonach die Olympischen Spiele eine Veranstaltung der kapitalistischen „Bourgeoisie“ seien, 
und strebte daher überhaupt keine Olympiateilnahme an. Für die Sportfunktionäre in den westlichen Besatzungszonen war die Wiederaufnahme in die Olympische Bewegung hingegen erklärtes Ziel. Die 1947 erfolgte Neugründung eines Deutschen Olympischen Ausschusses erkannte das IOC jedoch nicht an.

Erst mit der Gründung der Bundesrepublik Deutschland konnte die erneute „Rückkehr nach Olympia“ erfolgreich eingeleitet werden: Im Rahmen der Bundesfeier in Bonn wurde am 24. September 1949 ein Nationales Olympisches Komitee (NOK) gegründet. Erster Präsident wurde Herzog Adolf Friedrich zu Mecklenburg, 1951 übernahm Karl Ritter von Halt das Amt. Im selben Jahr wurde das deutsche NOK vom IOC anerkannt, die formalen Voraussetzungen für eine Olympiateilnahme 1952 waren somit erfüllt.

Als jedoch die DDR auf Geheiß der Sowjetunion - die ihre anti-olympische Haltung aufgegeben hatte und fortan ebenfalls an den Olympischen Spielen teilnehmen wollte - 1951 ein eigenes Nationales Olympisches Komitee unter dem Vorsitz von Kurt Edel (1920-1987) gründete, entstand eine deutsch-deutsche Gemengelage, die die internationale Sportpolitik in den folgenden Jahren immer wieder beschäftigen sollte (Spitzer, 1999). Im Vorfeld der NOK-Gründung in der DDR hatte man im Westen die eigene Institution in „NOK für Deutschland“ umbenannt, um so - im Einklang mit der Bundespolitik - den deutschen Alleinvertretungsanspruch zu untermauern. Das IOC, dem ab 1952 der US-Amerikaner Avery Brundage als Präsident vorstand, folgte dieser Auffassung und verweigerte dem DDR-NOK zunächst die Anerkennung. Die Offerte, unter bundesdeutscher Führung an den Olympischen Spielen 1952 teilzunehmen, lehnten die DDR-Sportfunktionäre trotz der zuvor erfolgten Unterzeichnung einer diesbezüglichen Vereinbarung zur Verärgerung des IOC ab.

Erst mit dem diplomatisch geschickter agierenden Heinz Schöbel (1913-1980) als neuem NOK-Präsidenten kam die DDR ihrem Ziel der eigenständigen Olympiateilnahme näher: Nach der 1955 erfolgten „provisorischen" NOK-Anerkennung durften DDR-Athletinnen und -Athleten ab 1956 an den Olympischen Spielen teilnehmen, zunächst allerdings nur im Rahmen einer gesamtdeutschen Mannschaft. Dieses bis 1964 fortgeführte Konstrukt - ein Zeichen des IOC, der politischen Realität der deutschen Teilung trotzen zu wollen - stellte für die Sportpolitik fortan eine große Herausforderung dar; nicht zuletzt, da mit Blick auf die Olympischen Spiele 1964 sogar der Abbruch der innerdeutschen Sportbeziehungen nach dem Bau der Berliner Mauer überwunden werden musste (Höfer, 1999). 
Abbildung 7: „Gesamtdeutsch nach Tokio“, Titelblatt „Der Spiegel“ vom 2. September 1964. Die Olympischen Spiele wurden vom 10. bis 24. Oktober 1964 in der japanischen Hauptstadt ausgetragen.

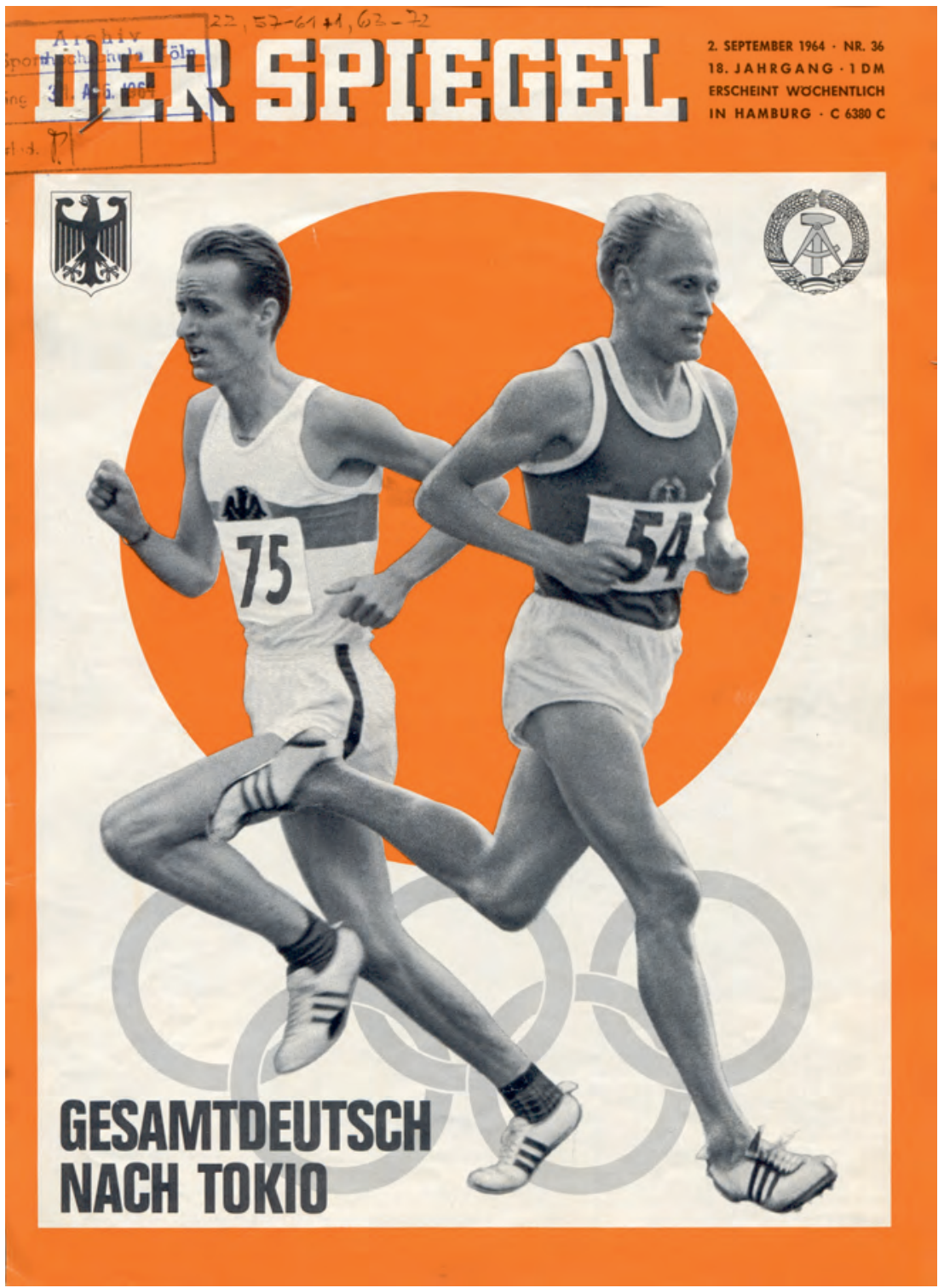

(Carl und Liselott Diem-Archiv/Zentrum für Olympische Studien, Deutsche Sporthochschule Köln) 
Nachdem das IOC der DDR 1965 schließlich die Eigenständigkeit zugestanden hatte, gingen bei den Olympischen Spielen 1968 erstmals getrennte deutsche Teams an den Start. Als verbindendes Element aus den Vorjahren waren allerdings noch die gemeinsame neutrale Fahne (Schwarz-Rot-Gold mit Olympischen Ringen) und die Hymne (Beethovens „Ode an die Freude“) geblieben.

Über diese "Querelle d'allemand“ hinaus prägten im Zeitalter des Kalten Kriegs globale politische Spannungen die Olympische Bewegung, zudem übertrug sich die Rassismusproblematik in verschiedenen Ländern auf die Olympischen Spiele - das „Black Power“-Bild der schwarzen USAthleten Tommie Smith (geb. 1944) und John Carlos (geb. 1945), die bei den Olympischen Spielen 1968 in Mexico-City nach dem Gewinn der Gold- beziehungsweise Bronzemedaille im 200m-Lauf auf dem Siegerpodest die Faust zum Protest gegen die Diskriminierung der afroamerikanischen Bevölkerung erhoben, wurde zur Foto-Ikone (Davis, 2018).

Weitere große Herausforderungen für die Olympische Bewegung stellten der zunehmende Einfluss der Medien - insbesondere des Fernsehens - und die Frage nach dem Fortbestand des Coubertin'schen Amateursportideals in einer sich stetig professionalisierenden Sportwelt dar.

\section{München 1972}

Für die bundesdeutschen Funktionäre bedeutete die 1965 auch auf der olympischen Bühne sichtbar gewordene deutsche Teilung eine Niederlage, die aber gleichzeitig zum Impuls wurde, ein sportpolitisches Zeichen zu setzen: Die bundesdeutschen Sportvertreter mit dem seit 1961 amtierenden NOK-Präsidenten Willi Daume (1913-1996) an der Spitze wollten München ins Rennen um die Austragung der Olympischen Spiele 1972 schicken. Hans-Jochen Vogel (1926-2020), damals Oberbürgermeister der bayerischen Landeshauptstadt, sagte noch im Oktober 1965 seine Unterstützung zu, auch Bundeskanzler Ludwig Erhard (1897-1977) begrüßte die Initiative. Im Dezember 1965 gab die Bundesregierung die vom IOC geforderten Garantien. Einen Tag vor dem Jahreswechsel 1965/66 wurde die Olympiabewerbung beim IOC eingereicht. Bei der Wahl im April 1966 setzte sich München gegen die Mitbewerberstädte Montreal, Madrid und Detroit durch.

Das Organisationskomitee für die Olympischen Spiele 1972 mit Willi Daume als Präsident wurde am 3. Juli 1966 gegründet, das Generalsekretariat unter der Leitung von Herbert Kunze (1908-2007) nahm am 1. Januar 1967 seine Arbeit auf. 
Insbesondere Daume schwebte ein olympisches Gesamtkunstwerk vor, das einen farbenfrohen und heiteren Charakter aufweisen sollte. So wollte man der internationalen Gemeinschaft ein gänzlich anderes Deutschland als das von 1936 zeigen. Zentrale Aufgabenfelder für das Organisationskomitee waren die Erstellung neuer, architektonisch moderner Sportstätten sowie eine von Freundlichkeit und Fröhlichkeit geprägte Inszenierung (Schiller \& Young, 2010). Große - und langfristige - Wirkung hinterließen die Arbeit des Architekten Günther Behnisch (1922-2010) mit seiner Idee einer Olympiaparklandschaft und das Werk des für die visuelle Gestaltung verantwortlichen Designers Otl Aicher (1922-1991). 
Abbildung 8: „Olympiapark München“, Broschüre des Organisationskomitees für die Olympischen Spiele 1972.

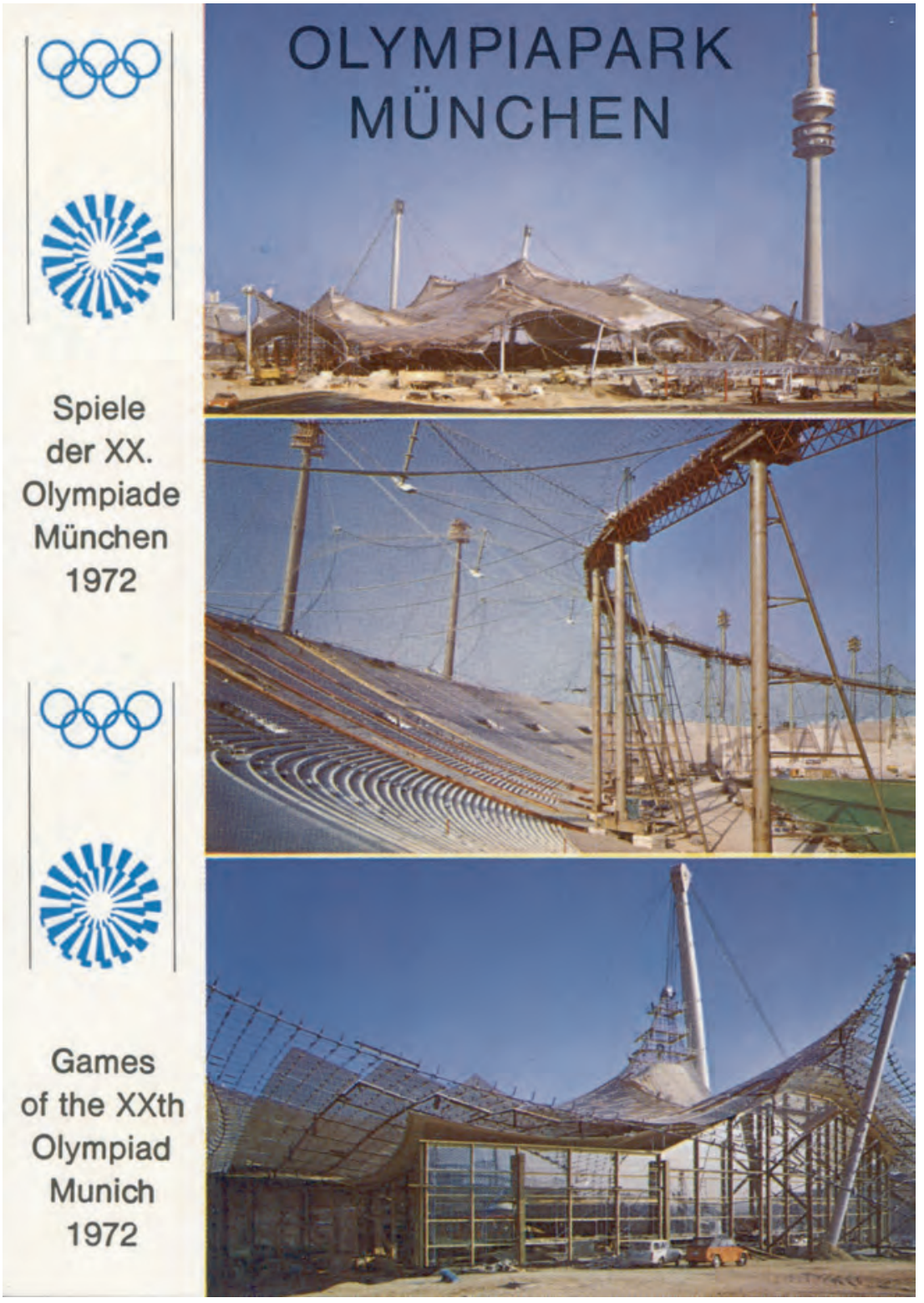

(Carl und Liselott Diem-Archiv/Zentrum für Olympische Studien, Deutsche Sporthochschule Köln) 
Gleichzeitig fand die Arbeit des Organisationskomitees aber auch vor dem Hintergrund des Kalten Kriegs statt, insbesondere die deutsch-deutsche Politik stellte eine Herausforderung dar: Für das Jahr 1972 stand der erste olympische Auftritt der DDR als souveräner Staat mit der Präsentation der eigenen Flagge und Hymne an - ein bis dahin auf bundesdeutschem Boden verbotener Akt. Dementsprechend symbolisch aufgeladen war vor allem die vor den Augen der Sportwelt veranstaltete Eröffnungsfeier am 26. August 1972.

Aber auch der sportliche Wettkampf wurde zunehmend in die politische Auseinandersetzung hineingezogen, die vermeintliche Überlegenheit des eigenen Systems sollte durch Siege belegt werden. Bereits 1968 in Mexiko-Stadt hatte das DDR-Team mehr Goldmedaillen gewonnen als die bundesdeutsche Auswahl, die im Vorfeld der Münchner Spiele intensivierte Leistungssportförderung führte zu noch größerer Dominanz der DDR in der bayerischen Landeshauptstadt und bei den folgenden Olympischen Spielen - und stellt bis heute ein weites Forschungsfeld zum Thema "Staatsdoping" dar.

Am 5. September 1972 traten jedoch sowohl die inszenatorische Leichtigkeit der Olympischen Spiele als auch der sportliche Wettstreit in den Hintergrund: Der Überfall der palästinensischen Terrorgruppe "Schwarzer September" auf die Mannschaft Israels im Olympischen Dorf endete in einem Blutbad, nach dem gescheiterten Befreiungsversuch auf dem Flughafen Fürstenfeldbruck waren elf ermordete israelische Athleten und Offizielle zu beklagen. Auch ein deutscher Polizist und fünf Geiselnehmer kamen zu Tode.

Das Attentat bedeutete das Ende der „heiteren Spiele“, nicht aber der Olympischen Spiele 1972. Nach einer zentralen Trauerfeier im Olympiastadion wurden die Wettkämpfe am 7. September 1972 - unter Zustimmung Israels - fortgesetzt, gemäß dem Satz „The Games must go on!“ des IOC-Präsidenten Avery Brundage, den dieser bei der Zeremonie am Vortag geäußert hatte (Schiller \& Young, 2010). 
Abbildung 9: „Die Welt trauert um die Opfer der Olympia-Mannschaft von Israel", Titelblatt des Bulletins des Presse- und Informationsamts der Bundesregierung vom 8. September 1972.

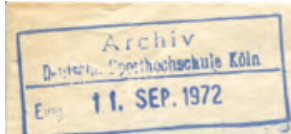

Presse- und Informationsamt der Bundesregierung

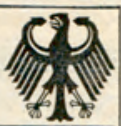

Nr. 122/S. 1521 Bonn, den 8. September 1972

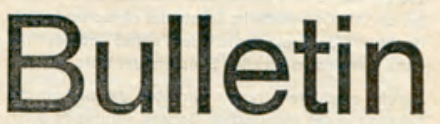

Die Welt trauert um die Opfer der Olympia-Mannschaft von Israel

Trauerfeier für die Toten von München

und Fürstenfeldbruck im Olympia-Stadion

Am 6. September 1972 gedachten die Teilnehmer aus aller Welt im Olympia-Stadion in München in einer Trauerfeier der Opfer des verbrecherischen Anschlags arabischer Terroristen.

Der Präsident des Organisationskomitees, Willi Daume, der Chef de Mission der israelischen Mannschaft, Shmuel Lalkin, der Botschafter des Staates Israel, Eliashiv Ben-Horin, der Präsident der Bundesrepublik Deutschland, Gustav W. Heinemann, und der Präsident des IOC. Avery Brundage, ehrten in Traueransprachen die Toten, verurteilten das bar. barische Verbrechen und bekundeten ihren Willen zur Brüderlichkeit und Versōhnung.

\section{Ansprache des OK-Präsidenten}

Der Prăsident des Nationalen Olympischen Komitees und Organisationskomitees fưr die Splele der XX. Olympiade, Willi Daume, gedachte der Opfer mit folgender Ansprache:

Trauergemeindel Für uns, die wir mit festem Vertrauen auf den guten Willen aller Menschen die Spiele der XX. Olympiade vorbereitet haben, ist dieser Tag ein Tag unendlicher Trauer.

Alles, was sich so schön zu erfülien schien, ein Fest, das bis gestern so sichtbar die Sehnsucht der Menschen nach Verstehen, Freude und Frieden zum Ausdruck brachte, ist durch menschliche Schuld ohnegleichen in Frage gestellt worden.

Selbst in der Welt des Verbrechens gibt es noch Tabus, gibt es eine letzte Schranke der Entmenschli- chung. vor der man zurückschreckt. Diese Schranke haben die Schuldigen im Olympischen Dorf durchbrochen. Sie sind mit Mord in das schōne, große Fest der Völker der Erde eingebrochen, in ein Fest, das dem Frieden gilt.

Die Härten und Gefahren des Irdischen werden nicht immer nach eigener Gunst der Enwartung verteilt.

Mōge aber wenigstens dieses Durchbrechen der letzten Schranken menschlicher Gesittung die Welt aufrütteln, endlich der Gewalttătigkeit zu entsagen, siè als menschenfeindlich und abscheulich zu verurteilen und zu verachten, wo immer und zu welchem Zweck sie auch angewendet wird.

Wir vereinen uns in der Traver mit den Familien, der Mannschaft, den Lãndern, indem wir den Tag mit seinen unreifen Rasereien hinter uns lassen. Und es gibt nur den Trost, dab wir nicht selbst unser Schicksal formen, sondern daß unsere Gegenwart und unsere Zukunft liegt in eines Höheren Hand.

\section{Inhalt}

Die Welt trauert um die Opfer

der Olympia-Mannschaft von Israel

OK-Präsident Will Daume

Chef de Mission Shmuel Lalkin

Botschafter Eliashiv Ben-Horin

Bundespräsident Gustay W. Heinemann $\quad 1523$

IOC-Prăsident Avery Brundage 1523

Erklärung des Bundeskanzlers

Bestürzung und Trauer

über den Anschlag in München

Bundeskanzler Willy Brand

20 Jahre deutsch-israelisches

Wiedergutmachungsabkommen
1521

1522 23 24 25

1527

(Carl und Liselott Diem-Archiv/Zentrum für Olympische Studien, Deutsche Sporthochschule Köln) 
Bekanntermaßen wurden die Olympischen Spiele auch in der Folgezeit fortgesetzt, an der Spitze des IOC stand seit 1972 Lord Michael Killanin (1914-1999). Die Spiele wurden jedoch mehr und mehr zum Spielball politischer Interessen. Insbesondere die umfassenden Olympiaboykotte der 1970er und 1980er Jahre wurden zur existenziellen Bedrohung. Entscheidende Impulse für die Neuausrichtung der Olympischen Bewegung gingen vom 1981 in Baden-Baden veranstalteten 11. Olympischen Kongress aus: Unter dem seit 1980 amtierenden IOC-Präsidenten Juan Antonio Samaranch (1920-2010) wurde das bis dahin strikt geltende Teilnahmeverbot für Profisportlerinnen und -sportler liberalisiert, nachdem sich die Aufrechterhaltung des traditionellen Amateurparagraphen als nicht mehr umsetzbar erwiesen hatte. Weiterhin wurde die Vermarktung der Olympischen Spiele beschlossen, das IOC mit seinem „Kapital“ aus den weltweit bekannten olympischen Ringen konnte sich in den folgenden Jahren zum Global Player entwickeln und die NOKs finanziell fördern.

\section{Literatur:}

Bernett, H., \& Teichler, H.J. (1999). Olympia unter dem Hakenkreuz. In M. Lämmer (Hrsg.), Deutschland in der Olympischen Bewegung - Eine Zwischenbilanz (S. 127-171). Frankfurt: NOK für Deutschland.

Coubertin, P. de (1931). Mémoires olympiques. Lausanne: Bureau Intern. de Pédagogie Sportive.

Davis, D. (2018). „That's my 15 minutes in the sun. An Interview with John Carlos. Journal of Olympic History. The official Publication of the International Society of Olympic Historians, 26 (2), 8-16.

Decker, W. (1996). Die Olympien des Evangelis Zappas. In Decker, W., Dolianitis. G., \& Lennartz, K. (Hrsg.), 100 Jahre Olympische Spiele. Der neugriechische Ursprung (S. 41-59). Würzburg: Ergon.

Diem, C. (1914). Friede zwischen Turnen und Sport. Leipzig/Berlin: Teubner.

Höfer, A. (1999). Querelle d'allemand. Die gesamtdeutschen Olympiamannschaften (1956-1964). In M. Lämmer (Hrsg.), Deutschland in der Olympischen Bewegung - Eine Zwischenbilanz (S. 209-259). Frankfurt: NOK für Deutschland.

Jönsson, Å. (2001). Nordiska spelen: historien om sju vinterspel $i$ Stockholm av olympiskt format 1901 till 1926. Stockholm: Diagolos.

Kluge, V. (1997). Olympische Sommerspiele. Die Chronik I, Athen 1896 - Berlin 1936. Berlin: Sportverlag. 
Kluge, V. (1999). Olympische Winterspiele. Die Chronik. Chamonix 1924 - Nagano 1998. (3. Auflage). Berlin: Sportverlag.

Langenfeld, H. (1999). Die ersten beiden Jahrzehnte. In M. Lämmer (Hrsg.), Deutschland in der Olympischen Bewegung - Eine Zwischenbilanz (S. 41-83). Frankfurt: NOK für Deutschland.

Lennartz, K. (1999). Die Zeit der Weimarer Republik. In M. Lämmer (Hrsg.), Deutschland in der Olympischen Bewegung - Eine Zwischenbilanz (S. 85-118). Frankfurt: NOK für Deutschland.

Molzberger, A. (2012). Zwischen Patriotismus und Internationalität: Die Olympischen Spiele 1912 in Stockholm. In M. Lämmer (Hrsg.), Studien zur Sportgeschichte. St. Augustin: Academia.

Molzberger, A., Wassong, S., Quanz, D., \& Sühl, R. (2018). Abgestaubt und neu erforschbar. Die historischen Sammlungen der Deutschen Sporthochschule Köln. Band 2: Olympische Bewegung. Hellenthal: Sportverlag Strauß.

Müller, N. (1994). Cent ans de Congrès Olympiques 1894-1994. Histoire - Objectifs Réalisations. Lausanne: Comité International Olympique.

Naul, R. (1999). Willibald Gebhardt - der „deutsche Coubertin“. In M. Lämmer (Hrsg.), Deutschland in der Olympischen Bewegung - Eine Zwischenbilanz (S. 3739). Frankfurt: NOK für Deutschland.

Schiller, K., \& Young, C. (2010). The 1972 Munich Olympics and the Making of modern Germany. Berkeley: Univ. of California Press.

Spitzer, G. (1999). Zwischen 1945 und 1952: Drei NOKs in Deutschland. In M. Lämmer (Hrsg.), Deutschland in der Olympischen Bewegung - Eine Zwischenbilanz (S. 177-203). Frankfurt: NOK für Deutschland. 